\title{
UTILIZING BRIGHTNESS TEMPERATURE HISTOGRAMS FOR MICROWAVE RADIOMETER HIGH FREQUENCY (150-183 GHZ) CALIBRATION
}

\author{
Rachael Kroodsma [rachael.a.kroodsma@nasa.gov] \\ ESSIC, University of Maryland / NASA Goddard Space Flight Center
}

\section{Introduction}

On-orbit calibration of spaceborne microwave radiometers is necessary to correct for:

- Solar intrusions

- Attitude offsets

- Calibration drifts

- Scan obstructions

- Intercalibration

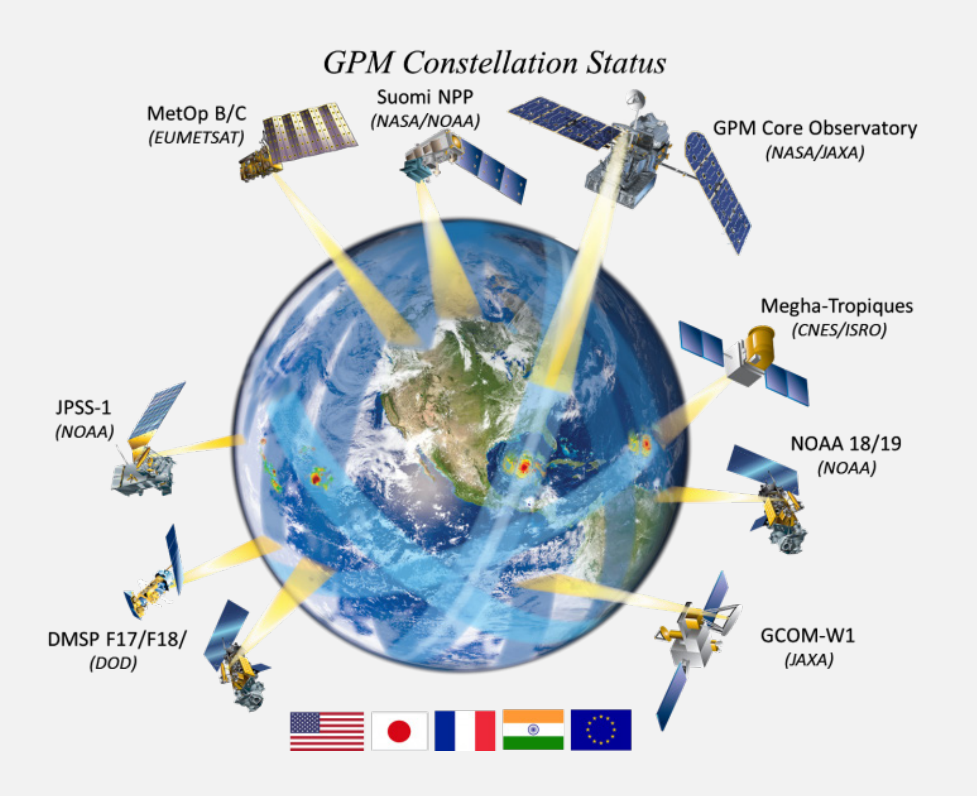

Utilizing several unique methods

for on-orbit calibration give confidence to results. This presentation describes a new method for $150-183 \mathrm{GHz}$ channels.

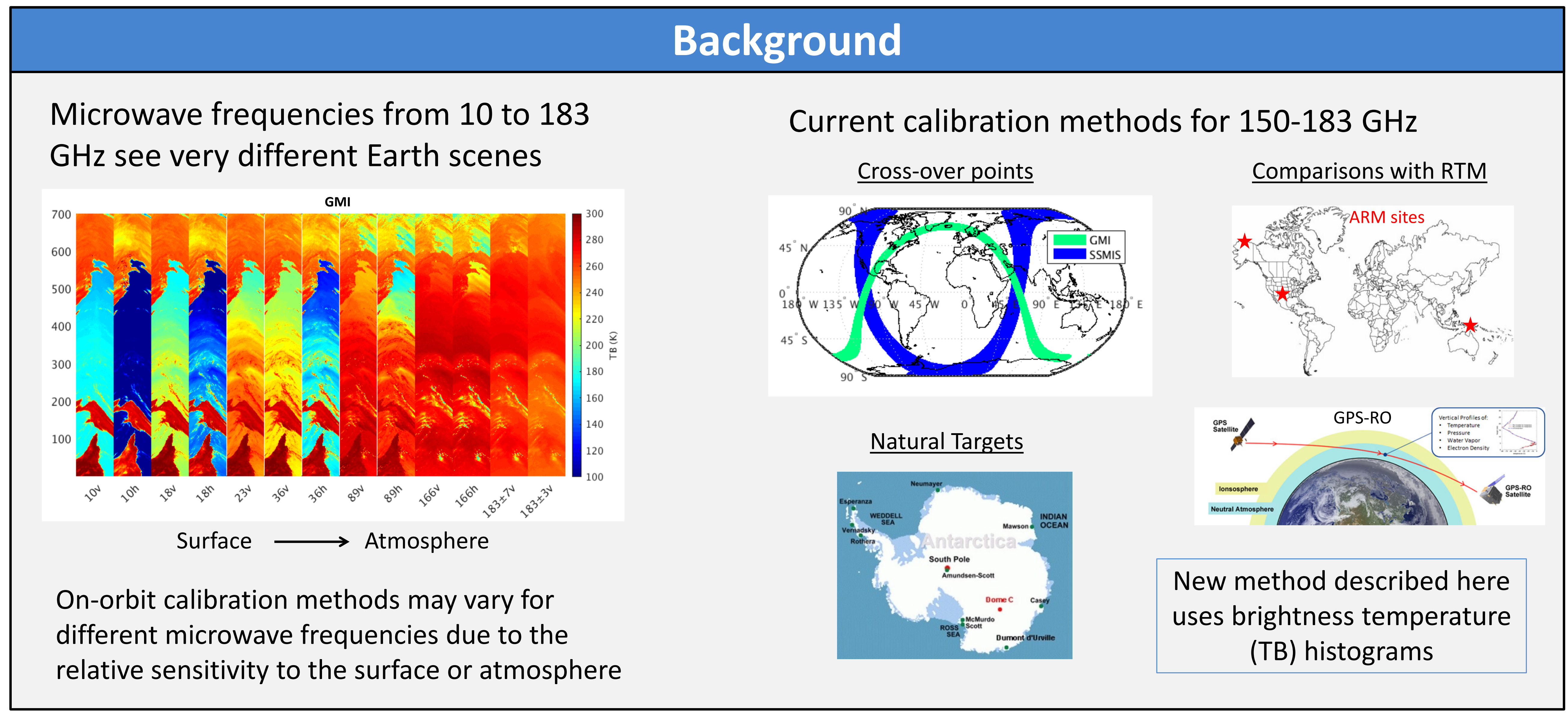

\section{Method}

GMI TB histograms for one month of observations GMI observed (obs) and simulated (sim) histograms
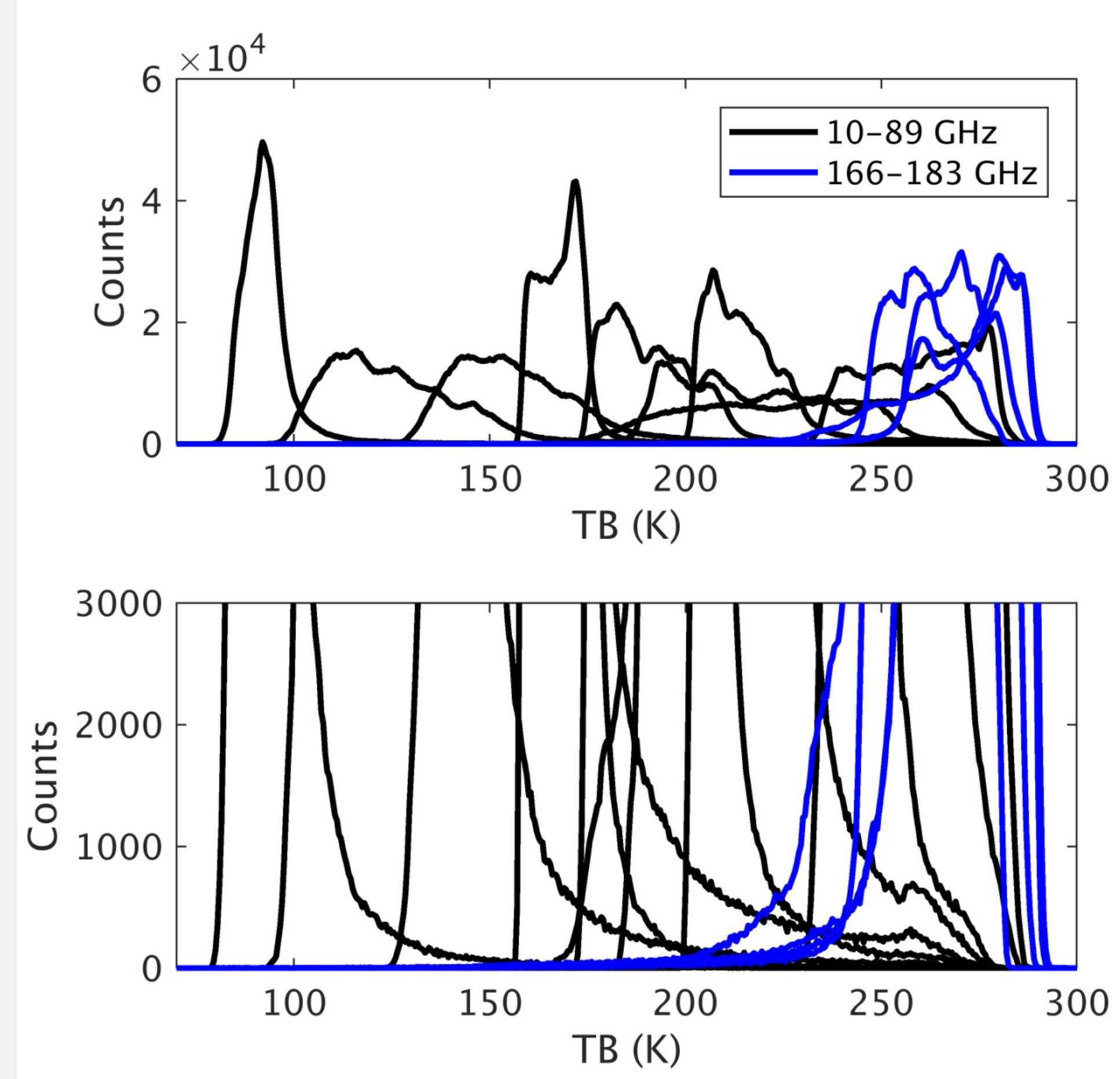

10-89 GHz: cold side of histogram has sharp edge 166-183 GHz: warm side of histogram has sharp edge

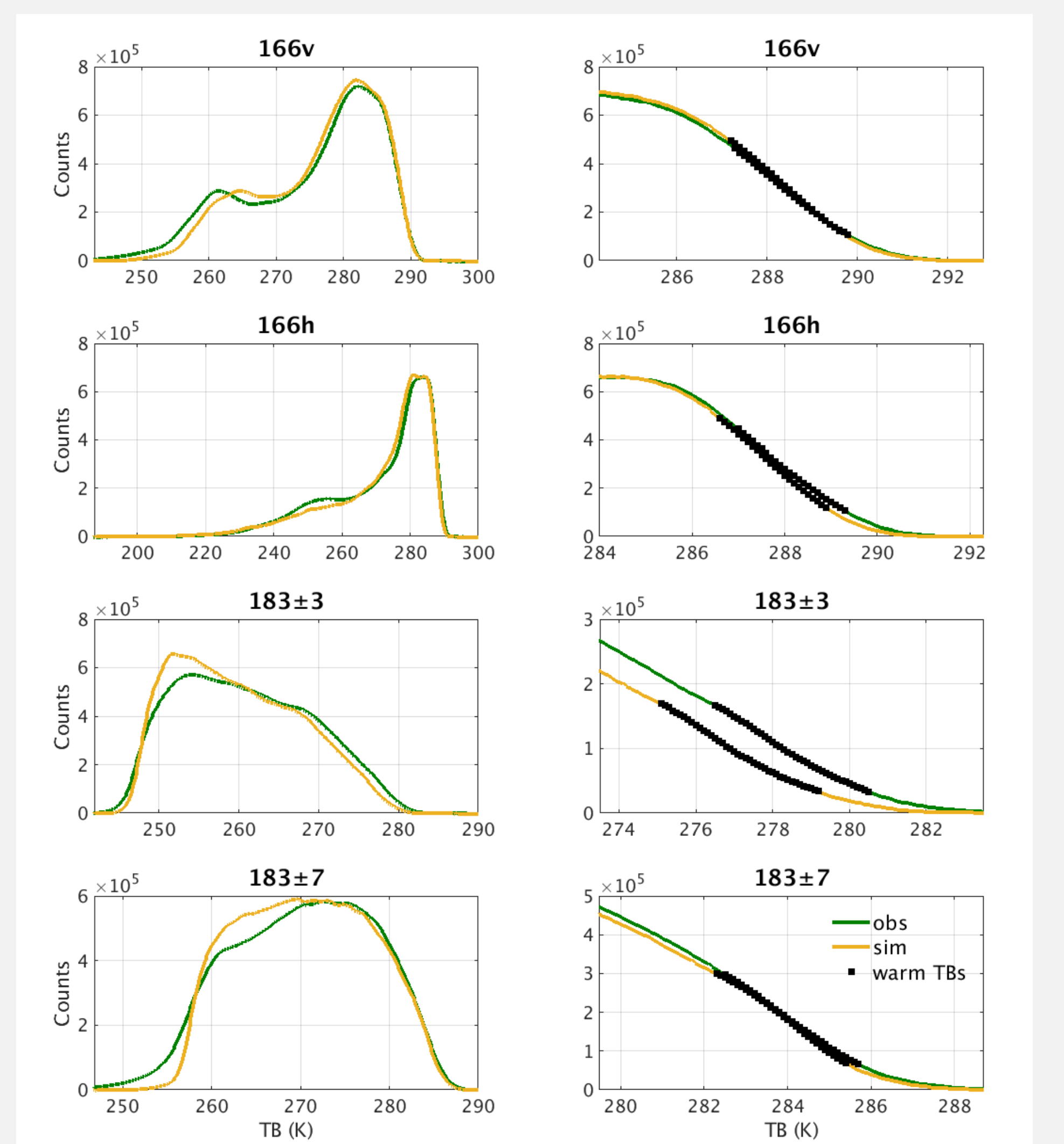

- Shapes of obs and sim histograms similar at warm end

- Black dots indicate portion of histogram used for calibration

- Use single difference (SD: obs - sim) to analyze radiometer calibration
Where do the warm TBs occur?
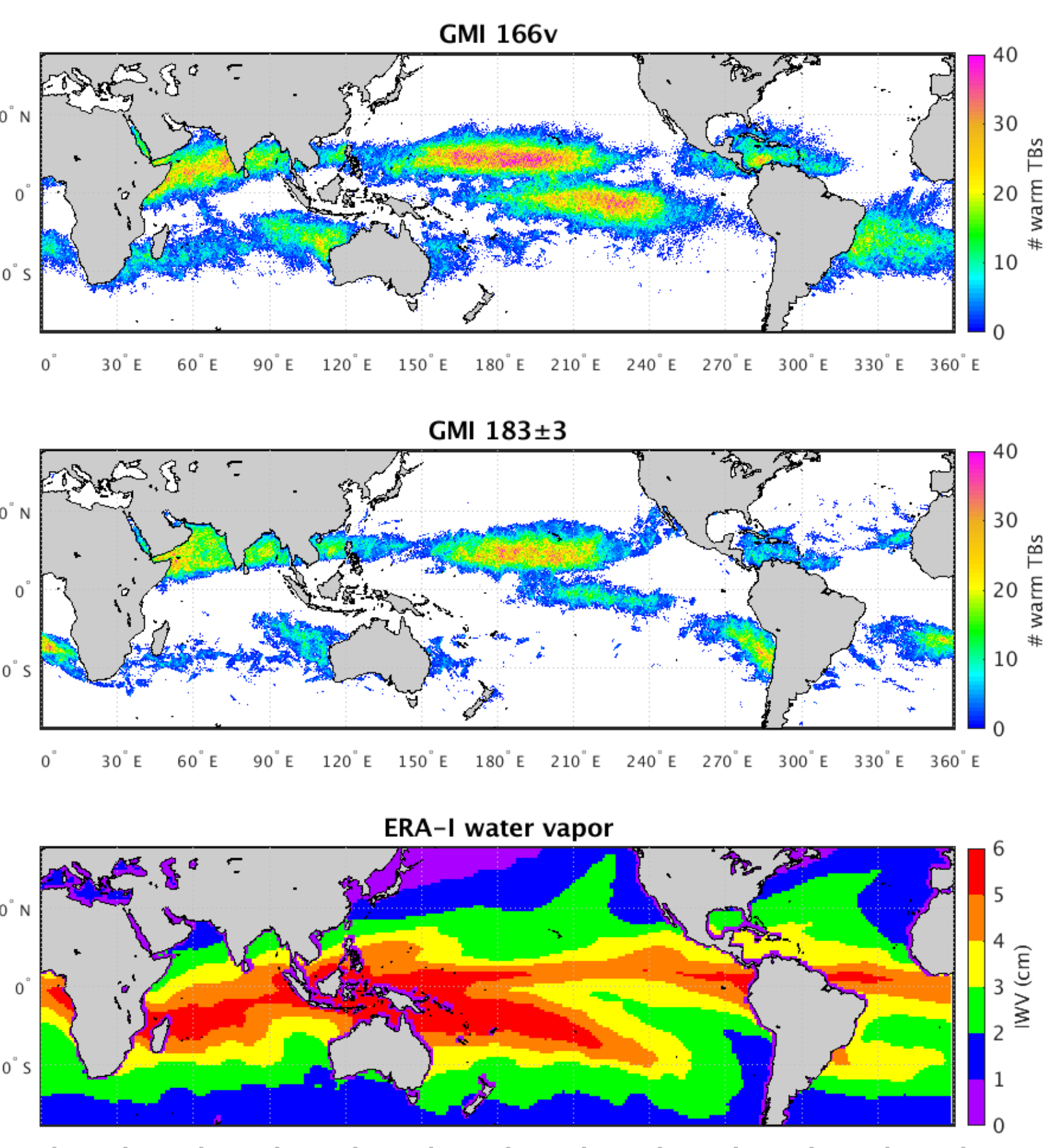

Warm TBs occur in tropical regions with minimal water vapor

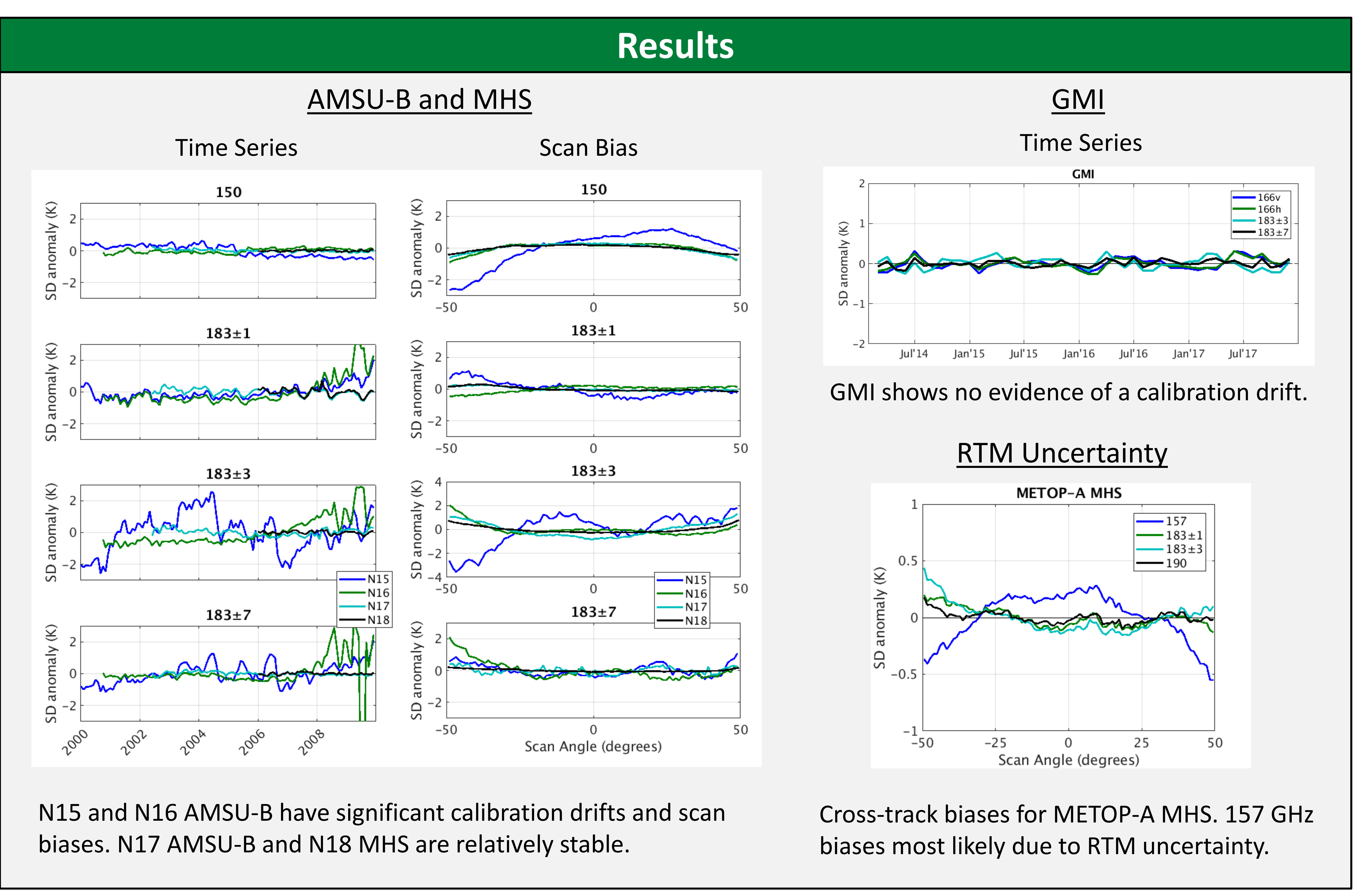

Summary

New on-orbit calibration method is presented for microwave radiometer 150-183 GHz channels

- Utilizes the shape of brightness temperature histograms

- Can be used in combination with other methods to corroborate results

- Does not require cross-overs between satellites or observations of a specific region

- Application to cross-track sounders and conical imagers show promising results

Future Work

- Mitigate impact of RTM on sounder crosstrack scan biases

- Improve intercalibration of similar but different channels (e.g. 150 with $166 \mathrm{GHz}$ )

\section{References}

